\title{
Sketched Graph Drawing: A Lesson in Empirical Studies
}

\author{
Helen C. Purchase \\ School of Computing Science, University of Glasgow, UK
}

\begin{abstract}
This paper reports on a series of three similar graph drawing empirical studies, and describes the results of investigating subtle variations on the experimental method. Its purpose is two-fold: to report the results of the experiments, as well as to illustrate how easy it is to inadvertently make conclusions that may not stand up to scrutiny. While the results of the initial experiment were validated, instances of speculative conclusions and inherent bias were identified. This research highlights the importance of stating the limitations of any experiment, being clear about conclusions that are speculative, and not assuming that (even minor) experimental decisions will not affect the results.
\end{abstract}

Keywords: graph sketching, empirical studies, replication, limitations.

\section{Introduction}

This paper reports on a series of three similar experiments with a common aim: to determine the graph drawing layout principles favored when participants draw graphs. The first experiment of the series was peer-reviewed and published in a reputable journal [1].

Typically in information visualization or HCI research, researchers run an experiment, form conclusions, publish, and then move on to the next interesting question. The second and third experiments reported here arose from a reflective critique of the first experiment. In this case, the experimenter (the author of this paper) did not "publish and move on", but explored subtle aspects of the experimental design, attempting to replicate and confirm the initial results.

The focus of this paper is therefore two-fold: to present the experiments and their results, but also to describe a process whereby revisiting empirical work has highlighted interesting facts about the process of conducting empirical studies.

This motivation for this paper relates to the idea of reflective critique, an idea borrowed from the practice of action learning projects. Action learning projects [2] do not aim to address specified research questions, and their approach is formative rather than summative, resulting in a cycle of continuous improvement. This approach is unlike the typical experimental research project, where a research question is clearly defined, a methodology is designed to address it, data is collected, and final conclusions made and published. In contrast, an action learning methodology encourages honest reflection on outcomes, and these reflections are fed into another cycle of the process.

This paper, therefore, reports on the results of two experiments conducted 'after the fact', i.e. after conclusions from the first experiment had been disseminated, with the latter two experiments being designed as a result of reflection and critique.

S. Wismath and A. Wolff (Eds.): GD 2013, LNCS 8242, pp. 232-243, 2013.

(C) Springer International Publishing Switzerland 2013 


\section{Background}

The design of automatic graph layout algorithms tend to be based on common 'aesthetic principles', for example, the elimination of edge crossings or the minimisation of adjacent edge angles. Early experimental research investigating how graphs may best be laid out tended to use a task-based performance approach (e.g. [345]), and established key findings such as the fact that a high occurrence of crossed edges reduced performance and prominent depiction of symmetry increased performance.

More recent empirical research has instead focussed on the manner in which participants create their own visual layout of relational information as a graph drawing. Van Ham and Rogowitz [6] (HR08) asked participants to adjust manually the layout of existing graph drawings. They used four graphs of 16 nodes, each with two clusters separated by one, two, three and four edges respectively. The graphs were presented in a circular and a spring layout [7], giving a total of eight starting diagrams, shown in random order. They collected 73 unique drawings, and found that most participants separated the two clusters, that the human drawings contained $60 \%$ fewer edge crossings than the automatically produced drawings, and that humans did not value uniform edge length as much as the spring algorithm did.

Dwyer et. al [8] (D+09) performed a similar hands-on experiment, asking participants to lay out two social networks, each with a circular initial arrangement. Participants were encouraged to lay the graphs out in a way that would best support the identification of cliques, chains, cut nodes and leaf nodes. With a focus on the process of layout rather than on the product, the only observation that they make about the graphs produced is that users removed edge crossings.

The first experiment in the series reported here (Experiment 1, [1]) was designed to address a similar research goal as HR08 and D+09, using a different methodology. The research question is Which graph drawing layout principles do people favour when creating their own drawings of graphs?

There are five main design features of Experiment 1 that differentiate it from HR08 and $\mathrm{D}+09$, the differentiating design features (DDFs):

DDF-1. The participants had to both draw the graph, as well as lay it out, a more complex task than both HR08 and D+09;

DDF-2. The participants drew the graphs from scratch, so were not biased by any initial layout (both HR08 and D+09 used an initial configuration);

DDF-3. A sketching tool was used, so the physical drawing process was unhindered by an intermediate editing interface;

DDF-4. Video data was collected, so both the process and product of creation were able to be analysed (this was done by D+09, but not HR08);

DDF-5. Layout preferences were discussed with the participants in a post-experiment interview (this was done by D+09, but not HR08).

Four graphs were used, two practise graphs and two experimental graphs. Data on product, process and preferences were collected 1 .

\footnotetext{
${ }^{1}$ Note that the publication arising from Experiment 1 also included graphs drawn in a pointand-click mode, but only the sketched graph experiment is considered here.
} 
The several conclusions of Experiment 1 as published [1] included:

CONC-1 The layout principles that participants favoured during the process of laying out their drawings were often not evident in the final product.

CONC-2 The principle of fixing nodes and edges to an underlying unit grid was prominent.

\subsection{Reflective Critique: Issues Arising}

After peer-review, publication, presentation, and independent citation of this first experiment and its results, audience members at two seminars suggested some subtle variations on the experimental method: not new research questions, simply small amendments. As is typical in such situations, this author responded that such variations could be addressed as part of 'future work'.

It was not necessary to investigate these issues (the paper had already been published after all), but they led the experimenter to reflect on the research, and to question to what extent results might be biased by a method.

Three issues arose as a result of this reflection:

- If participants compromise their layout design during creation of the drawing (CONC-1), does this mean that they are not happy with their final product? Experiment 2 addressed the issue of whether participants were satisfied with their final drawing, or whether they expressed disappointment that they were unable to conform to their desired layout.

- If participants favour a grid-based layout (CONC-2), would they prefer a drawing laid out using an algorithm that aligns nodes and edges to an underlying grid to their own? Experiment 2 investigated whether the participants preferred their own sketched graph drawing to a similar one that conformed to a grid layout.

- Was the tendency to favour a grid layout and straight lines (CONC-2) a consequence of the way in which the graph information stimuli were presented as a list of edges? Experiment 3 investigated whether representing the graph structure in an alternative text format also produces results that favour grid layout.

\section{The Experiments}

\subsection{Experimental Process for all Three Experiments}

The primary research question in all three of these graph sketching experiments is: Which graph drawing layout principles do people favour when creating their own drawings of graphs? Participants were asked to draw graphs and their drawings were analysed for evidence of common graph drawing layout principles.

Equipment: A graph-drawing sketch tool, SketchNode [9] was used, allowing nodes, edges and node labels to be sketched, edited and moved with a stylus on a tablet screen, laid flat. 
Task: Participants were given a textual description of a graph and asked to draw it in SketchNode, with the instruction to Please draw this graph as best as you can so to make it "easy to understand". They were deliberately not given any further instruction as to what "easy to understand" means, nor primed with any information about common graph layout principles (e.g. minimising edge crossings, use of straight edges, etc,). They had as long as they liked to draw and adjust the layout of the graphs.

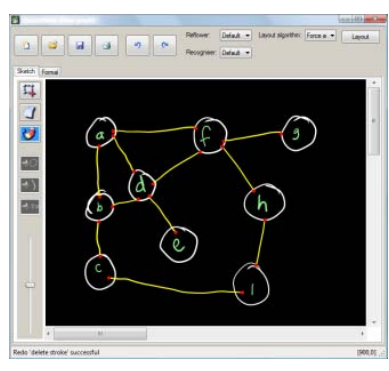

Graphs: Two experimental graphs were used in all three experiments: graph A (10 nodes, 14 edges) and graph B (10 nodes, 18 edges).

Experimental method: Participants completed required ethical procedures and provided demographic information. All the relevant features of SketchNode were demonstrated, and participants were given the opportunity to practice and ask questions. Two practice graphs ensured participants were comfortable with the task and system.

The two experimental graphs A and B, were then presented to the participants, with the graph edges presented in different random order for each participant, and counterbalanced between participants. At the end of the experiment, the participants were asked "Why did you arrange the graphs in the way you did?" in a recorded interview.

The participants: Participants in all three experiments were of a similar profile: a mixture of students and non-students, of both sexes, with around half the student participants in each experiment studying some form of computer science.

\subsection{Differences between the Experiments}

In Experiment 1, the most important differentiating feature in comparison with prior research was the way the graphs were presented (DDF-2). HR08 and D+09 presented their graphs as graph drawings which already had some layout properties (circular and spring in HR08 and circular in D+09). So as not to bias the participants towards any layout principles, the graphs in Experiment 1 were presented in textual form, as a list of edges (Table 1 column 1).

Experiments 2 and 3 addressed four issues arising from Experiment 1.

Compromised layout: CONC-1 suggests participants may not have been entirely happy with their final drawing, as they had been obliged to compromise their favoured layout as the graph became more complex. Experiment 2 investigated the extent of this compromise, and whether participants acknowledged it.

The first research question for this experiment was "Do participants like the layout of their final product?" We speculated they would express dissatisfaction with their final product. Once they had drawn their graph, we asked them to indicate on a scale of 1-5 how "happy" they were with their drawing. 
Preference for a grid: CONC-2: Experiment 1 suggested a grid layout was favoured; we anticipated participants would prefer a grid layout to their own.

The second research question was "Do participants prefer their sketched drawing to be laid out in a grid format to their own layout?"

Experiment 2 used the two automatic graph layout algorithms in SketchNode: spring (based on Fruchterman and Reingold [10]) and grid (placing nodes and edges on the lines and vertices of an underlying unit grid). After layout, the visual sketched appearance of the nodes and labels remains the same, so the resultant diagram can be directly compared with original sketched drawing.

At the end of the sketching stage of Experiment 2, the participant's own drawing was laid out using these two algorithms. Participants were asked to rank the three drawings according to their preference.

In an attempt to eliminate any personal bias or recency effects, a willing subset of the participants chose between hand-drawn and the two automatically laid out drawings two weeks after the experiment.

Validation: As both changes to the experimental method for Experiment 2 were postexperiment activities, Experiment 2 also served as a means to validate the results of Experiment 1.

Effect of graph format: One of the main differentiating features between Experiment 1 and prior research was that the graph information was presented in abstract form, rather than as a graph drawing (DDF-2), and the participants drew the graphs from scratch. Here we investigated whether even this abstract form had produced a bias.

The research question for this experiment was "Does the format in which the graph structure is represented affect the layout of the sketched graph drawings produced?" For Experiment 3, we presented the graphs as an adjacency list (Table 1, column 3), and followed exactly the same process as Experiment 1. This format is visually quite different from the simple list of edges, as each edge is not clearly specified as an individual pair, and it is more obvious which nodes have a higher degree. We wished to investigate whether a format that does not focus on the individual node pairs (as in Experiment 1) would still result in user-sketched drawings that conform to a grid structure.

Table 1 shows the differences between the experiments, as well as those factors that remained the same. Figure 1 shows example sketches from all three experiments.

\section{Data Analysis}

Compromised layout (Experiment 2): The participants in Experiment 2 indicated how happy they were with their own sketches (five-point scale, 5=perfectly happy). Graph A's mean: 4.14, graph Bs' mean: 3.5; both graphs together: 3.91.

Participants were asked what they didn't like about their drawings, and how they would improve them. None mentioned that they would have liked to conform to a grid layout; most comments related to local issues like the size and shape of the nodes, and connections between the nodes and edges. The few comments that referred to overall layout of the drawing were concerned with spreading the nodes out, symmetry and circular layout. There were also several comments about the need to plan in advance. 
Table 1. Summary of the differences between the three experiments

\begin{tabular}{|c|c|c|c|}
\hline & Experiment 1 & Experiment 2 & Experiment 3 \\
\hline experimental graphs & $\begin{array}{l}\mathrm{A}(n=10, e=14) \\
\mathrm{B}(n=10, e=18)\end{array}$ & as Experiment 1 & as Experiment 1 \\
\hline experimental task & $\begin{array}{l}\text { After introduction and } \\
\text { training activities, } \\
\text { participants sketched } \\
\text { the two graphs }\end{array}$ & as Experiment 1 & as Experiment 1 \\
\hline equipment & SketchNode & as Experiment 1 & as Experiment 1 \\
\hline number of participants & 17 & 22 & 26 \\
\hline $\begin{array}{l}\text { form of graph presented } \\
\text { to participants }\end{array}$ & $\begin{array}{l}(A, D)(A, C)(B, D) \\
(C, D)(B, C)(B, E) \\
(C, E)(E, J)(F, G) \\
(J, F)(F, I)(G, I) \\
(J, H)(I, H)\end{array}$ & as Experiment 1 & \begin{tabular}{l|l} 
S & U R \\
Q & S \\
V & W Z \\
Y & X V \\
R & U \\
W & Y \\
U & Z \\
T & R Q S \\
X & Z
\end{tabular} \\
\hline $\begin{array}{l}\text { post-experiment } \\
\text { discussion }\end{array}$ & none & $\begin{array}{l}\text { participants indicated } \\
\text { how happy they were } \\
\text { with their drawing }\end{array}$ & as Experiment 1 \\
\hline $\begin{array}{l}\text { post-experiment } \\
\text { ranking }\end{array}$ & none & $\begin{array}{l}\text { participants ranked } \\
\text { sketched drawings } \\
\text { against associated } \\
\text { spring and grid } \\
\text { drawings }\end{array}$ & as Experiment 1 \\
\hline
\end{tabular}

Preference for a grid (Experiment 2): Once participants had sketched their graph, the two graph layout algorithms (Section 3.2 above) were applied to their drawing.

A three-way-set (TWS) is a set of three drawings: a participant's sketched drawing, and two versions of this sketch produced by the algorithms. Each participant has a TWS-GA and TWS-GB. Figure 2 shows a TWS for one of the participant's graphs.

Participants ranked the drawings in their own TWS-GA and TWS-GB at the end of Experiment 2. After two weeks, we contacted all participants for a follow-up ranking experiment; fourteen took part. They ranked their own TWS-GA and TWS-GB (as before), as well as the TWS-GA and TWS-GB for two other participants. They were not told their own drawings were included in these sets (Table 2).

The data were analysed with Friedman tests with adjusted pairwise comparisons. The only significant results related to graph B (highlighted in Table 2):

\footnotetext{
2 The notational convention is: graph A drawn by participant 3 is $3 \mathrm{~A}$; graph $\mathrm{B}$ drawn by participant number 8 is $8 \mathrm{~B}$. The suffix $-1,-2$ or -3 indicates experiment 1,2 or 3 .
} 


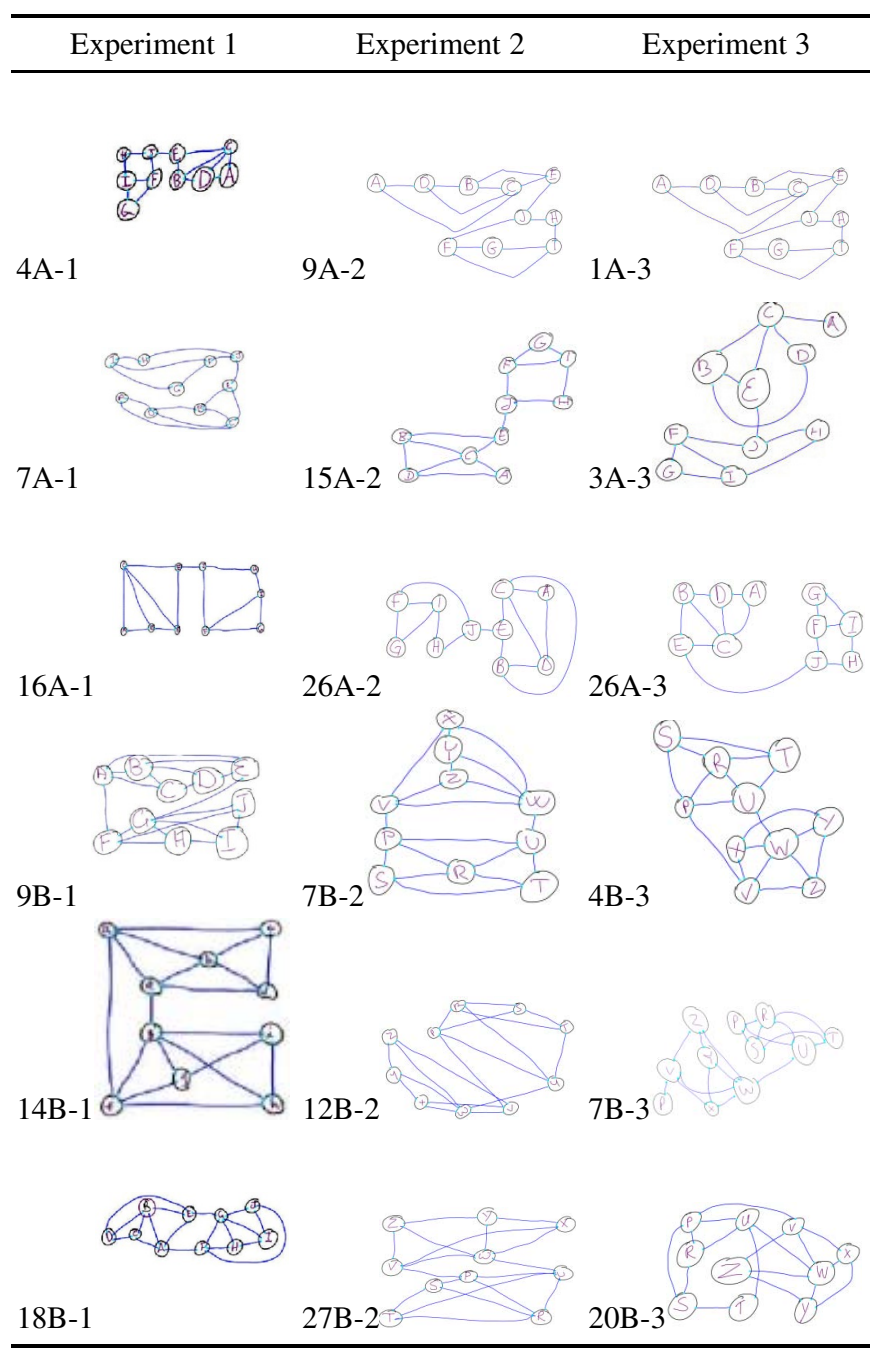

Fig. 1. Example drawings from all three experiment 2
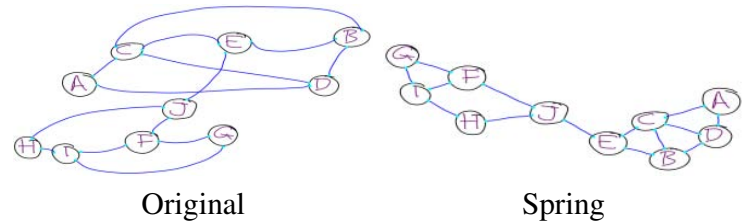

Spring

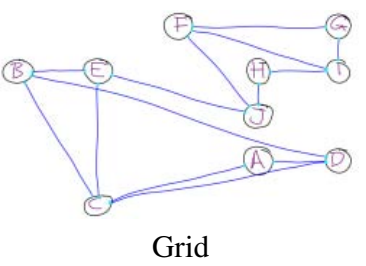

Fig. 2. The TWS-GA for participant 17. Note that the hand-drawn nature and form of the nodes and their labels is retained. 
Table 2. Mean ranking of TWSs ( $3=$ most preferred). Significant results in italics.

\begin{tabular}{lllllll}
\hline & $\begin{array}{l}\text { number of } \\
\text { participants : } \\
\text { TWSs }\end{array}$ & $\begin{array}{l}\text { mean } \\
\text { rank: } \\
\text { sketch }\end{array}$ & $\begin{array}{l}\text { mean } \\
\text { rank: } \\
\text { spring }\end{array}$ & $\begin{array}{l}\text { mean } \\
\text { rank: } \\
\text { grid }\end{array}$ & $\begin{array}{l}\% \text { times } \\
\text { sketch } \\
\text { preferred }\end{array}$ & $\begin{array}{l}\% \text { times } \\
\text { grid } \\
\text { preferred }\end{array}$ \\
\hline $\begin{array}{l}\text { After drawing the graph } \\
\text { (own TWS) }\end{array}$ & $22: 22$ & GA 2.45 & 1.77 & 1.77 & $50 \%$ & $23 \%$ \\
& & GB 2.54 & 1.41 & 2.05 & $68 \%$ & $23 \%$ \\
\hline Two weeks later (own TWS) & \multirow{2}{*}{$14: 14$} & GA 2.07 & 1.86 & 2.07 & $36 \%$ & $29 \%$ \\
& \multirow{2}{*}{$\begin{array}{l}\text { Two weeks later (own + two } 1.79 \\
\text { other TWSs) }\end{array}$} & GA 2.00 & 1.79 & 2.43 & $29 \%$ & $43 \%$ \\
\hline
\end{tabular}

- immediately after the experiment, the participants' ranking for their own sketched graph was higher than that for the spring layout $(p<0.001)$;

- two weeks after the experiment, the participants' ranking for the grid layout was higher than the spring layout $(\mathrm{p} ; 0.001)$, and higher than their own drawing $(\mathrm{p}=$ 0.019 ) in the set of three sketched graphs that included their own.

Validation (Experiment 2): Experiment 1 found that participants appeared to favour a grid layout and horizontal and vertical edges in their sketched graph drawings. We analysed the 44 graph drawings from Experiment 2 for the following key layout features (Table 3):

- Number of edge crossings: points outside of the node boundaries where one or more edges cross.

- Number of straight lines. A visual assessment as to whether an edge was intended to be straight was agreed by two independent coders.

- Number of vertical or horizontal edges, and grid structure. A visual assessment was agreed by two independent coders as to whether edges were intended to be horizontal or vertical, and whether drawings had been drawn with a grid in mind.

Independent samples two-tailed t-tests were conducted using the 34 drawings from Experiment 1 and the 44 drawings from Experiment 2 (Table 3).

Effect of graph format (Experiment 3): We analysed the 52 graph drawings from Experiment 3 using the same layout features as for Experiments 1 and 2.

Independent samples two-tailed t-tests were conducted using the 34 drawings from Experiment 1 and the 52 drawings from Experiment 3 were used (Table 3).

In the interviews, as before, no participants spoke directly of a grid layout; there were comments about local features (size of the nodes, the need for straight lines), and layout (crossings, symmetry, distance between nodes). When asked why they drew the graph as they did, many said the adjacency list itself suggested the structure of the drawing: they 'worked from top to bottom.' 
Table 3. Comparing key layout features in the graphs produced in the experiments.

\begin{tabular}{|c|c|c|c|c|c|c|c|}
\hline & \multirow[t]{2}{*}{$\begin{array}{c}\text { E1 } \\
n=34\end{array}$} & \multirow[t]{2}{*}{$\begin{array}{c}\mathrm{E} 2 \\
n=44\end{array}$} & \multirow[t]{2}{*}{$\begin{array}{l}\text { E3 } \\
\imath=52\end{array}$} & \multicolumn{2}{|c|}{$\begin{array}{l}\text { validation: } \\
\text { E1 v. E2 }\end{array}$} & \multicolumn{2}{|c|}{$\begin{array}{l}\text { effect of format: } \\
\text { E1 vs E3 }\end{array}$} \\
\hline & & & & $t$ & $p$ & $t$ & $p$ \\
\hline Number of crosses/drawing & 2.21 & 4.05 & 2.38 & 1.410 & 0.163 & 0.216 & 0.829 \\
\hline $\begin{array}{l}\text { Number of crosses/drawing (excl. } \\
\text { outliers) }\end{array}$ & 1.24 & 2.05 & 1.62 & 1.400 & 0.166 & 0.776 & 0.440 \\
\hline$\% \mathrm{HV}$ edges & $33.6 \%$ & $28.0 \%$ & $23.3 \%$ & 1.232 & 0.222 & 2.842 & $0.006^{a}$ \\
\hline$\%$ straight edges & $88.1 \%$ & $85.8 \%$ & $90.3 \%$ & 0.472 & 0.638 & 0.541 & 0.590 \\
\hline Drawn with grid in mind & $10(29 \%)$ & $7(16 \%)$ & $4(8 \%)$ & 1.433 & 0.156 & 2.478 & $0.007^{b}$ \\
\hline
\end{tabular}

${ }^{a}$ More HV edges in Experiment 1. ${ }^{b}$ More grids in Experiment 1

\section{Results}

Compromised layout: We wished to see whether participants expressed any dissatisfaction with their drawings as a result of having to compromise the layout while creating the drawing: a stated conclusion of Experiment 1.

In general, the participants were satisfied with the layout of their own drawings: their satisfaction ratings were high; they expressed little dissatisfaction, and did not mention that they would have liked to have been able to conform to a grid.

This is an instance of stating a conclusion based on insufficient data. In Experiment 1 , observation of the videos (by two independent coders) suggested that participants conformed to a grid in the initial stages of drawing, but that this layout feature was abandoned later in the drawing process. This conclusion was, however, simply suggestive, and there was no qualitative interview data to back it up. It appears that, even if layout compromises had been made, participants were not aware of having done so.

Preference for a grid: We wished to see whether participants would prefer a grid-based layout to their own layout.

While initially they preferred their own drawings in Experiment 2, when the recency effect of having just drawn the graph was eliminated, the grid layout was ranked as substantially better than the sketches for the more complex graph. However, none of the participants mentioned a grid formation or horizontal or vertical edges in their comments.

It appears that participants know what they like when they see it (and when it is not in competition with a drawing that they know is their own), but cannot independently articulate the layout features that contribute to what they like.

This is an instance of stating an incorrect conclusion based on qualitative data. Despite the Experiment 1 drawings being analyzed by two independent coders who formed the same conclusion (that participants preferred a grid), this inferred conclusion does not hold when more direct data is collected.

Validation: The fact that there are no significant differences in the values for the key metrics for Experiment 2 suggests validation of the results of Experiment 1: when the 
graph is presented as a list of edges, participants tend to favour a grid layout and straight lines.

This is an instance of validating data. Using identical methodologies for experiments 1 and 2, we would expect similar values for the dependent data variables.

Effect of graph format: We wished to see whether the form in which the graph stimuli are presented would affect the results.

The drawings produced in Experiment 3 from adjacency list stimuli did not favour a grid layout; there is a significant difference on the key metrics of horizontal and vertical edges, and grid formation, between Experiments 1 and 3.

The format in which the relational graph information is presented to the participants thus affects the final layout of their drawing, a result echoing experimental results on visual metaphors [11]. The participants spoke of 'following the table from top to bottom' in Experiment 3; it is likely that the participants in Experiment 1 followed the edge list from left to right.

There is an irony here: Experiment 1 presented the graph as a list of edges so to address possible layout biases in HR08 and D+09, who presented graphs as drawings. It seems even using a simple list of edges can introduce a bias.

This is an instance of unintended bias. Even a simple (and seemingly innocuous) decision in Experiment 1 introduced a factor that biased the results.

Table 4. Summary and comparison of the findings for the three experiments

\begin{tabular}{llll}
\hline & Experiment 1 & Experiment 2 & Experiment 3 \\
\hline compromised layout & $\begin{array}{l}\text { assumed, from video } \\
\text { data }\end{array}$ & not proven & not investigated \\
\hline $\begin{array}{l}\text { preferring a grid } \\
\text { over own drawing }\end{array}$ & $\begin{array}{l}\text { assumed, from sketch } \\
\text { graph drawing data }\end{array}$ & $\begin{array}{l}\text { some support after } \\
\text { elimination of recency } \\
\text { effects }\end{array}$ & not investigated \\
\hline validation & not applicable & $\begin{array}{l}\text { validation of key graph } \\
\text { drawing metrics }\end{array}$ & not appropriate \\
\hline $\begin{array}{l}\text { effect of graph } \\
\text { format }\end{array}$ & not considered & not investigated & effect found \\
\hline
\end{tabular}

\section{Discussion}

\subsection{Implications for this Research}

Our speculation that participants prefer the result of a grid-based algorithm over their own drawing was partially confirmed, but only when the 'personal pride' factor had diminished over time, and for the more complex graph. It is still clear, however, that while participants might prefer a grid layout in both creation and recognition, they are less able to articulate this preference.

The most surprising result is that how the graph is presented to the participants has a significant effect on the form of their drawing — this was something that had not been 
considered originally. It suggests that the only way bias may be eliminated entirely would be by asking the participant to draw a graph based on their internal cognitive structure, and not on an externally visible form. This may mean describing a scenario to the participant while attempting to avoid verbal bias (for example, a social network) and then asking the participant to draw the graph representing the relational information.

Of course, this story could not simply end here, as there are several outstanding issues to address about all three of these experiments. Do these results extend to larger graphs? What would happen if the participants were all novice computer users? Or if a digital whiteboard were used? Of if participants were told that the graphs related to a domain (e.g. a transport network or a circuit diagram)? Or if they were explicitly advised to plan in advance? Further experiments would no doubt shed more light on these initial studies (and would probably reveal some unexpected results).

\subsection{Implications for Experimental Research}

The results of our investigation of subtle experimental variations suggest that:

- There will always be a bias relating to the manner in which information is presented to an experimental participant;

- Even if the results of an experiment are validated by repeating it, these results may still be compromised by bias;

- Firm experimental conclusions need validation through replication and multiple sources of data.

We did not set out to investigate the effect of experimental subtleties: our original aim was not to run a series of comparative experiments. If it had been, then we might have conducted a broader experiments-within-an-experiment study, preferably using the same participants throughout, and followed a systematic process of enquiry. No: we initially set out to investigate what happens when participants draw graphs from scratch - and we published a peer-reviewed paper in a reputable journal presenting the results of this study, as is common practice: researchers run an experiment, collect data, form conclusions, and publish. And then typically move on to their next experiment.

The contribution of this paper is therefore broader than the simple 'run an experiment and report' model: by reflecting on and critiquing our own experimental work, and investigating issues arising from the critique, we have demonstrated the limitations of this common practice.

It is rare that researchers repeat an experiment with subtle variations - doing so has revealed that there is still much to learn about the nature of user-sketched graphs, that even a carefully-conducted experiment may have flaws, that there is value in not simply moving on to the next 'big' question, and that repeating an experiment so as to investigate subtleties may produce surprising results. All experiments have limitations - no experiment can ever be perfect. This paper demonstrates the importance of acknowledging these limitations, of validating results where possible, and of reading published experimental results with healthy critical attitude.

Acknowledgments. SketchNode is the result of significant efforts of Beryl Plimmer and Hong Yul, of the University of Auckland. The experiments were conducted by 
Christopher Pilcher, Rosemary Baker, Anastasia Giachanou, and Gareth Renaud. John Hamer assisted with data analysis, coding and editing. Ethical approval was given by the Universities of Auckland and Glasgow.

\section{References}

1. Purchase, H.C., Pilcher, C., Plimmer, B.: Graph drawing aesthetics — created by users not algorithms. IEEE Trans. Vis. and Computer Graphics 18(1), 81-92 (2012)

2. Kemmis, S., McTaggart, R.: The Action Research Planner, 3rd edn. Deakin University Press (1988)

3. Huang, W.: Using eye-tracking to investigate graph layout effects. In: Hong, S.H., Ma, K.L. (eds.) Proc. Asia Pacific Symp. on Visualisation, pp. 97-100. IEEE (2007)

4. Ware, C., et al.: Cognitive measurements of graph aesthetics. Information Visualization 1(2), 103-110 (2002)

5. Purchase, H.C., Cohen, R.F., James, M.: An experimental study of the basis for graph drawing algorithms. ACM J. Experimental Algorithmics 2(4), 1-17 (1997)

6. van Ham, F., Rogowitz, B.E.: Perceptual organisation in user-generated graph layouts. IEEE Trans. Vis. and Computer Graphics 14(6), 1333-1339 (2008)

7. Gansner, E.R., Koren, Y., North, S.C.: Graph Drawing by Stress Majorization. In: Pach, J. (ed.) GD 2004. LNCS, vol. 3383, pp. 239-250. Springer, Heidelberg (2005)

8. Dwyer, T., et al.: A comparison of user-generated and automatic graph layouts. IEEE Trans. on Vis. and Computer Graphics 15(6), 961-968 (2009)

9. Plimmer, B., Purchase, H.C., Yang, H.Y.: SketchNode: Intelligent sketching support and formal diagramming. In: Brereton, M., Viller, S., Kraal, B. (eds.) OzChi Conference, Brisbane, Australia, pp. 136-143 (2010)

10. Fruchterman, T.M.J., Reingold, E.M.: Graph drawing by force-directed placement. Software — Practice and Experience 21(11), 1129-1164 (1991)

11. Ziemkiewicz, C., Kosara, R.: The shaping of information by visual metaphors. IEEE Trans. on Vis. and Computer Graphics 14(6), 1269-1276 (2008) 